\title{
Mathematics Education as a Design Science
}

\author{
Richard Lesh $\cdot$ Bharath Sriraman
}

(C) FIZ Karlsruhe 2007

Erratum to: ZDM Mathematics Education (2005) 37(6)

DOI 10.1007/BF02655858

Unfortunately, due to publisher mistake the spelling of the author's name was incorrect in the online version. The name should read Bharath Sriraman.

The online version of the original article can be found under doi:10.1007/BF02655858.

R. Lesh

School of Education, Indiana University,

Bloomington, Indiana 47405-1006, USA

e-mail: ralesh@indiana.edu

B. Sriraman $(\bowtie)$

The Montana Mathematics Enthusiast, Department of Mathematical Sciences,

The University of Montana,

Missoula, MT 59812, USA

e-mail: sriramanb@mso.umt.edu

URL: http://www.montanamath.org/TMME 